\title{
A limit model for thermoelectric equations
}

\author{
Luisa Consiglieri*
}

\begin{abstract}
We analyze the asymptotic behavior corresponding to the arbitrary high conductivity of the heat in the thermoelectric devices. This work deals with a steady-state multidimensional thermistor problem, considering the Joule effect and both spatial and temperature dependent transport coefficients under some real boundary conditions in accordance with the Seebeck-Peltier-Thomson cross-effects. Our first purpose is that the existence of a weak solution holds true under minimal assumptions on the data, as in particular nonsmooth domains. Two existence results are studied under different assumptions on the electrical conductivity. Their proofs are based on a fixed point argument, compactness methods, and existence and regularity theory for elliptic scalar equations. The second purpose is to show the existence of a limit model illustrating the asymptotic situation.
\end{abstract}

Keywords: Seebeck-Peltier-Thomson effect; Joule effect; Nemytskii operator

MSC2010: 35J25; 35Q60; 35Q79; 35D30

\section{Introduction}

In the recent years, the mathematical study of the thermistor problem has been of interest by various authors (see for instance [1, 9, 11, 30, 34] and the references therein) however the cross effects are neglected. Here we study a mathematical model for thermoelectric conductors, introduced in [10], taking into account the presence of the Seebeck-Peltier-Thomson and the Joule

*Independent Researcher Professor, Portugal. http://sites.google.com/site/luisaconsiglieri 
effects. Indeed, in the thermodynamics analysis, the Joule effect is given by $|\mathbf{j}|^{2} / \sigma(\cdot, \theta)$ with (cf. [25])

$$
\mathbf{j}=-\sigma(\cdot, \theta)(\alpha(\cdot, \theta) \nabla \theta+\nabla \phi)
$$

representing the current density, $\theta$ denoting the temperature, $\phi$ is the electric potential, and the electrical conductivity $\sigma$ is a known positive function. The electrical conductivity is assumed temperature dependent and this is different at different places along the material due to the molecular structure.

The Seebeck coefficient $\alpha$ is a given nonlinear function, dependent both in space and temperature, with constant sign observing that the sign of the Seebeck coefficient corresponds to the sign of the Hall effect [22]. The Thomson effect is $-\partial \alpha / \partial T(\cdot, \theta) \theta \nabla \theta \cdot \mathbf{j}$, where $\partial / \partial T$ means the derivative with respect to the real variable. Due to the first Kelvin relation, $\pi(\theta)=\theta \alpha(\theta), \theta \alpha(\theta)$ corresponds to the Peltier coefficient. Due to the second Kelvin relation, $\mu(\theta)=\theta \alpha^{\prime}(\theta)$, this coefficient is known as Thomson coefficient. Although $\mu$ is the only thermoelectric coefficient directly measurable for individual materials [18, 23], and the Seebeck coefficient appears as $\alpha(T)=\int_{T_{r}}^{T} \frac{\mu(t)}{t} d t$ for some reference temperature $T_{r}$, we keep the Seebeck coefficient as a given function as it is usual in the literature for the thermistor problem when this cross-effect coefficient is taken into account [2, 8, 33]. Thus, the resulting PDE's system is strongly coupled.

The thermoelectric problem under study reads (see its derivation in [10, 25])

$(\mathcal{P})$ Find the pair temperature-potential $(\theta, \phi)$ such that

$$
\begin{aligned}
-\nabla \cdot(k \nabla \theta)=\sigma(\cdot, \theta) \alpha(\cdot, \theta)\left(\alpha(\cdot, \theta)+\frac{\partial \alpha}{\partial T}(\cdot, \theta) \theta\right)|\nabla \theta|^{2}+ & \\
+\sigma(\cdot, \theta)\left(2 \alpha(\cdot, \theta)+\frac{\partial \alpha}{\partial T}(\cdot, \theta) \theta\right) \nabla \theta \cdot \nabla \phi+\sigma(\cdot, \theta)|\nabla \phi|^{2}+g & \text { in } \Omega, \\
-\nabla \cdot(\sigma(\cdot, \theta) \nabla \phi)=\nabla \cdot(\sigma(\cdot, \theta) \alpha(\cdot, \theta) \nabla \theta) & \text { in } \Omega, \\
k \nabla \theta \cdot \mathbf{n}=-\alpha(\cdot, \theta) \theta h & \text { on } \partial \Omega, \\
\sigma(\cdot, \theta)(\nabla \phi+\alpha(\cdot, \theta) \nabla \theta) \cdot \mathbf{n}=h & \text { on } \partial \Omega,
\end{aligned}
$$

where $\Omega$ is a convex bounded domain of $\mathbb{R}^{n}(n \geq 2)$, and it may represent electrically conductive rigid solids such as for instance thermistors, thermocouples, resistive thermal devices (also called resistance temperature detectors) or thermoelectric coolers (see [3, 5, 35] and the references therein). 
Notice that the boundary $\partial \Omega$ is Lipschitz since every bounded convex open subset of $\mathbb{R}^{n}$ always has a Lipschitz boundary [19, Section 1.2].

Here $\mathbf{n}$ is the unit outward normal to the boundary $\partial \Omega, g$ denotes the external heat sources and $h$ denotes the surface current source. The thermal conductivity $k$ is the known positive coefficient of the Fourier law. Finally, we remark that (4) is known as the linear Newton law of cooling with $\alpha(\theta) h$ representing the heat transfer coefficient.

Our two main purposes are: (1) to improve the existence result stated in [10] for three dimensional space, i.e. the solution belongs to the Sobolev space $W^{1, p}(\Omega)$ with $p>n=3$. Indeed, we state the existence result for the multidimensional case since its proof is valid for any $n \geq 2 ;(2)$ to pass to the limit on the thermal conductivity, $k \rightarrow+\infty$, in order to show the existence of the limit model

$$
\begin{aligned}
& \nabla \cdot(\sigma(\cdot, \Theta) \nabla \phi)=0 \quad \text { in } \Omega \\
& \sigma(\cdot, \Theta) \nabla \phi \cdot \mathbf{n}=h \quad \text { on } \partial \Omega
\end{aligned}
$$

for some positive constant $\Theta$ solving an implicit scalar equation. The nonlocal problem (6) -(7) is known as the shadow system, the heuristic designation introduced by Nishiura 31] in order to exhibit minimal dynamics displaying the mechanism of basic pattern formation. Here the shadow system turns out the dynamic relation among the trivial rest states (constant solutions) and the large amplitude voltages. The present result generalizes the homogeneous Neumann boundary value problem already studied for nonlocal elliptic problems [7].

The regularity assumptions about the domain could be weakened if different techniques are provided (see [4, 13, 14, 17, 28, 36] and the references therein). We refer to [12] the existence of singularities of electromagnetic fields at corners and edges of a bounded Lipschitz domain with piecewise plane boundary.

The contents of this work are as follow. In next Section we state the assumptions and the main results. Section 3 deals with existence and regularity results for auxiliary problems. We prove in Section 4 the existence result for $n \geq 2$ when the electrical conductivity is assumed be a uniformly continuous function, and we obtain in Section 5 its limit model. The proof of the existence result valid for $n=2$ when the electrical conductivity is assumed be discontinuous on the space variable and the corresponding asymptotic limit model are postponed in Section 6. 


\section{Assumptions and main results}

In order to establish the existence results we assume the following set of hypotheses on the data.

(H1) $k$ is a positive constant.

(H2) $\sigma: \Omega \times \mathbb{R} \rightarrow \mathbb{R}$ is a Carathéodory function, i.e. measurable with respect to $x \in \Omega$ and continuous with respect to $T \in \mathbb{R}$, and furthermore

$$
\exists \sigma_{\#}, \sigma^{\#}>0: \quad \sigma_{\#} \leq \sigma(\cdot, T) \leq \sigma^{\#}, \quad \forall T \in \mathbb{R}, \text { a.e. in } \Omega .
$$

(H3) $g$ belongs to the Lebesgue space $L^{p / 2}(\Omega), p>2$, and $h \in C(\partial \Omega)$ is such that verifies the compatibility condition

$$
\int_{\partial \Omega} h d s=0
$$

where $d s$ represents the element of surface area.

(H4) $\alpha \in C(\bar{\Omega} \times \mathbb{R})$ is such that

$$
\exists L_{\alpha}>0: \quad\left|\alpha\left(x_{1}, T_{1}\right)-\alpha\left(x_{2}, T_{2}\right)\right| \leq L_{\alpha}\left(\left|x_{1}-x_{2}\right|+\left|T_{1}-T_{2}\right|\right),
$$

for all $x_{1}, x_{2} \in \bar{\Omega}$ and for all $T_{1}, T_{2} \in \mathbb{R}$, with $|x|$ representing the euclidean norm and $|T|$ the absolute value of a real number. Moreover, for all $x \in \Omega$, the mapping $T \mapsto \alpha(x, T)$ is continuously differentiable in $\mathbb{R}$ and its derivative satisfies

$$
\exists \mu^{\#}>0: \quad\left|\frac{\partial \alpha}{\partial T}(x, T)\right| \leq \begin{cases}\mu^{\#}, & \text { if }|T| \leq 1 \\ \mu^{\#} /|T|, & \text { if }|T|>1 .\end{cases}
$$

The following two different cases will be addressed:

$(\mathrm{H} 4)_{+}$for materials with positive $\alpha$ :

$$
\exists \alpha_{\#}, \alpha^{\#}>0: \quad \alpha_{\#} \leq \alpha(x, T) \leq \alpha^{\#}, \quad \forall x \in \bar{\Omega}, \quad \forall T \in \mathbb{R} .
$$

Moreover, there exist an open subset $\Gamma \subset \partial \Omega$ such that meas $(\Gamma)>$ 0 and meas $(\partial \Omega \backslash \bar{\Gamma})>0$ and constants $h_{\#}>0$ and $h_{1}<0$ such that

$$
h_{1}>-\frac{\min \left\{k, \alpha_{\#} h_{\#}\right\}}{C_{1} \alpha^{\#}}, \quad h(x) \geq \begin{cases}h_{\#}, & \text { a.e. } x \in \Gamma \\ h_{1}, & \text { a.e. } x \in \Sigma,\end{cases}
$$


where $\Sigma:=\partial \Omega \backslash \bar{\Gamma}$ and $C_{1}$ denotes the continuity constant of the embedding $H^{1}(\Omega) \hookrightarrow L^{2}(\Sigma)$, i.e.

$$
\|\theta\|_{2, \Sigma}^{2} \leq C_{1}\left(\|\nabla \theta\|_{2, \Omega}^{2}+\|\theta\|_{2, \Gamma}^{2}\right)
$$

for every $\theta \in H^{1}(\Omega)$;

(H4) - for materials with negative $\alpha$ :

$$
\exists \alpha_{\#}, \alpha^{\#}>0: \quad-\alpha^{\#} \leq \alpha(x, T) \leq-\alpha_{\#}, \quad \forall x \in \bar{\Omega}, \quad \forall T \in \mathbb{R} .
$$

Moreover, there exist an open subset $\Gamma \subset \partial \Omega$ such that meas $(\Gamma)>$ 0 and meas $(\partial \Omega \backslash \bar{\Gamma})>0$ and constants $h_{\#}>0$ and $h_{1}<0$ such that

$$
h_{1}>-\frac{\min \left\{k, \alpha_{\#} h_{\#}\right\}}{C_{1} \alpha^{\#}}, \quad h(x) \leq \begin{cases}-h_{\#}, & \text { a.e. } x \in \Gamma \\ -h_{1}, & \text { a.e. } x \in \Sigma .\end{cases}
$$

REMARK 2.1 In particular, 14) holds for the unity function then we have

$$
|\Sigma| \leq C_{1}|\Gamma|
$$

where $|\Gamma|=\operatorname{meas}(\Gamma)$ and $|\Sigma|=\operatorname{meas}(\partial \Omega \backslash \bar{\Gamma})$.

\section{Some remarks on the assumptions.}

1. The heat conductivity can be an uniformly continuous function on both variables verifying

$$
\exists k_{\#}, k^{\#}>0: \quad k_{\#} \leq k(x, T) \leq k^{\#}, \quad \forall x \in \Omega, \quad \forall T \in \mathbb{R} .
$$

Since our purpose is to study the asymptotic behavior as $k \rightarrow \infty$, we assume it as constant.

2. The case $\Gamma=\partial \Omega$ is excluded, from the fact that the Gauss theorem yields the necessary condition (9) of the existence of a solenoidal function satisfying (11) and (5) (cf. (3) ).

3. From the assumption (111) we obtain that $\left|\frac{\partial \alpha}{\partial T}(x, T)\right| \leq \mu^{\#}$, for all $x \in \Omega$ and for all $T \in \mathbb{R}$, and that the Thomson coefficient is bounded, i.e. $|\mu(x, T)|=\left|T \frac{\partial \alpha}{\partial T}(x, T)\right| \leq \mu^{\#}$, for all $x \in \Omega$ and for all $T \in \mathbb{R}$. 
4. By the Weierstrass Theorem, any continuous function defined on a compact set (of $\mathbb{R}^{n+1}$ ) is bounded. Then the upper bound in (12) as well as the lower bound in (15) could be given reduced to $|T|>1$.

We define the Nemytskii operators

$$
\sigma(\theta)=\sigma(\cdot, \theta(\cdot)) ; \quad \alpha(\theta)=\alpha(\cdot, \theta(\cdot)) ; \quad \frac{\partial \alpha}{\partial T}(\theta)=\frac{\partial \alpha}{\partial T}(\cdot, \theta(\cdot)),
$$

that map $L^{1}(\Omega)$ into $L^{q}(\Omega)$, for all $q<\infty$. Their designation is kept in order to clarify the presentation.

We endow the Sobolev space $W^{1, p}(\Omega), p>1$, with the equivalent norms:

- for the temperature solution

$$
\|\theta\|_{1, p, \Omega}=\|\nabla \theta\|_{p, \Omega}+\|\theta\|_{p, \Gamma}
$$

- for the potential solution

$$
\|\phi\|_{1, p, \Omega}=\|\nabla \phi\|_{p, \Omega},
$$

considering the correspondent Poincaré inequality.

Definition 2.1 We say that $(\theta, \phi)$ is a weak solution to (2)-(5) if $(\theta, \phi) \in$ $W^{1, p}(\Omega)^{2}$, for $p>n$, and it satisfies

$$
\begin{array}{r}
k \int_{\Omega} \nabla \theta \cdot \nabla \eta d x+\int_{\partial \Omega} \alpha(\theta) h \theta \eta d s= \\
=\int_{\Omega} \sigma(\theta)\left(\alpha(\theta)\left(\alpha(\theta)+\frac{\partial \alpha}{\partial T}(\theta) \theta\right)|\nabla \theta|^{2}+\left(2 \alpha(\theta)+\frac{\partial \alpha}{\partial T}(\theta) \theta\right) \nabla \theta \cdot \nabla \phi\right) \eta d x \\
+\int_{\Omega}\left(\sigma(\theta)|\nabla \phi|^{2}+g\right) \eta d x, \quad \forall \eta \in W^{1, p^{\prime}}(\Omega) ; \\
=-\int_{\Omega} \sigma(\theta) \alpha(\theta) \nabla \theta \cdot \nabla \eta d x+\int_{\partial \Omega} h \eta d s, \quad \forall \eta \in W^{1, p^{\prime}}(\Omega),
\end{array}
$$

where $p^{\prime}$ denotes the conjugate exponent to $p, p^{\prime}=p /(p-1)$. 
REMARK 2.2 If $\Omega$ is of class $C^{0,1}$ and $m p>n$, then the Morrey-Sobolev embedding holds

$$
W^{m, p}(\Omega) \hookrightarrow C^{0, m-n / p}(\bar{\Omega}) .
$$

The vector field $\mathbf{n}$ belongs only to $\mathbf{L}^{\infty}(\partial \Omega)$ if it is the unit outward normal vector to the boundary of $C^{0,1}$ domains. When $p>n$, the embedding $W^{1, p^{\prime}}(\Omega) \hookrightarrow L^{p n /(p n-n-p)}(\Omega)$ is valid. Thus the $L^{p / 2}$ behavior of the quadratic terms $|\nabla \theta|^{2}, \nabla \theta \cdot \nabla \phi,|\nabla \phi|^{2}$ is meaningful on the right hand side of (2) since $p / 2>p n /(p+n)$.

Let us extend the existence results whose can be found in [10]. The first main theorem states the existence of weak solutions to the problem under study, strengthening the assumption (H2), i.e. strengthening the regularity on $\sigma$.

Theorem 2.1 Assume $n \geq 2$, (H1) and (H3)-(H4) hold. Additionally we assume that $\sigma: \Omega \times \mathbb{R} \rightarrow \mathbb{R}$ is a uniformly continuous function satisfying (8) and the smallness condition (33) is satisfied. Then the variational problem (18)-(19) admits a weak solution $(\theta, \phi) \in W^{2, p n /(p+n)}(\Omega) \times W^{1, p}(\Omega)$, with $p>n$, in the sense of Definition 2.1, such that $\int_{\partial \Omega} \phi d s$. Moreover, the following estimates hold

$$
\begin{aligned}
\|\nabla \phi\|_{2, \Omega} \leq & \frac{1}{\sigma_{\#}}\left(\sigma^{\#} \alpha^{\#}\|\nabla \theta\|_{2, \Omega}+C_{2}\|h\|_{2, \partial \Omega}\right) ; \\
\|\theta\|_{1, p, \Omega} \leq & C_{p} \\
k\|\nabla \theta\|_{2, \Omega}^{2} \leq & C_{3}\left(\alpha^{\#}\left|h_{1}\right| C_{p}^{2}+\right. \\
& \left.+\left(A C_{p}^{2}+B C_{p}\|\nabla \phi\|_{2, \Omega}+\sigma^{\#}\|\nabla \phi\|_{2, \Omega}^{2}+\|g\|_{p / 2, \Omega}\right) C_{p}\right),
\end{aligned}
$$

where $C_{2}$ denotes the Poincaré-Sobolev continuity constant of the embedding $H^{1}(\Omega) \hookrightarrow L^{2}(\partial \Omega), C_{3}$ denotes the continuity constant of the embedding $W^{1, p}(\Omega) \hookrightarrow C(\bar{\Omega})$,

$$
A:=\sigma^{\#} \alpha^{\#}\left(\alpha^{\#}+\mu^{\#}\right), \quad B:=\sigma^{\#}\left(2 \alpha^{\#}+\mu^{\#}\right) ;
$$

and $C_{p}$ denotes a positive constant independent on $k$ if $k>\alpha_{\#} h_{\#}$.

If we assume $\sigma \in C(\bar{\Omega} \times \mathbb{R})$ as in [10], the Nemytskii operator maps $C(\bar{\Omega})$ into $C(\bar{\Omega})$ which implies the uniform continuity on the spatial variable.

Next, we establish the existence of a solution $\phi=\phi(\Theta)$ to (6) -(7) where $\Theta$ is solution of an implicit scalar equation. 
Theorem 2.2 Under the assumptions of Theorem 2.1, there exist weak solutions $\left(\theta_{k}, \phi_{k}\right) \in W^{2, p n /(p+n)}(\Omega) \times W^{1, p}(\Omega)$, with $p>n$, to the variational problem (18)-(19), such that

$$
\theta_{k} \rightarrow \Theta \quad \text { in } H^{1}(\Omega), \quad \phi_{k} \rightarrow \phi \text { in } H^{1}(\Omega),
$$

with $\Theta$ solving the implicit scalar equation

$$
\Theta \int_{\partial \Omega} \alpha(\cdot, \Theta) h d s=\int_{\Omega} \sigma(\cdot, \Theta)|\nabla \phi|^{2} d x+\int_{\Omega} g d x
$$

and $\phi$ solving (6)-(7). Moreover

$$
\frac{\sigma_{\#} \int_{\Omega}|\nabla \phi|^{2} d x+\int_{\Omega} g d x}{\alpha^{\#} \int_{\partial \Omega}|h| d s} \leq \Theta \leq \frac{\sigma^{\#} \int_{\Omega}|\nabla \phi|^{2} d x+\int_{\Omega} g d x}{\alpha_{\#} h_{\#}|\Gamma|+\alpha^{\#} h_{1}|\Sigma|} .
$$

REMARK 2.3 In face of (9) if the Seebeck coefficient is only a function on the temperature then (24) reads

$$
0=\int_{\Omega} \sigma(\cdot, \Theta)|\nabla \phi|^{2} d x+\int_{\Omega} g d x
$$

The presence of a generic heat source invalids the limit model (6)-(7) connected with the original thermoelectric problem introduced in [10].

The above results can be proved if the convexity of $\Omega$ is replaced by weaker assumptions, for instance when $\Omega$ is a plane bounded domain with Lipschitz and piecewise $C^{2}$ boundary whose angles are all convex [19, p. 151], or when $\Omega$ is a plane bounded domain with curvilinear polygonal $C^{1,1}$ boundary whose angles are all strictly convex [19, p. 174]. For general bounded domains $\Omega$ of $\mathbb{R}^{n}$ with Lipschitz boundary, it is known that the integrability exponents for the gradients of the potential and temperature solutions may be larger than 3 [17, 36], if the restriction to the case of uniformly continuous coefficients in (18)-(19) is assured. However, a generalization for such nonsmooth domains of Theorem 2.1 and its limit model is not a direct consequence. Indeed, new proofs will be needed because the compact embedding $W^{2, p n /(p+n)}(\Omega) \hookrightarrow \hookrightarrow$ $W^{1, p}(\Omega)$ is crucial to provide the weak continuity of the operator in the fixed point argument.

For the two-dimensional limit model, let us show the existence result under the minimal regularity on $\sigma$. 
Theorem 2.3 Assume $n=2$ and (H1)-(H4) hold. Then the problem (国)-(或) has a weak solution in the sense of Definition 2.1, for some $p>2=n$, under sufficiently small data.

Proposition 2.1 Under the assumptions of Theorem 2.3, there exists $\phi$ solving (6)-(7) with $\Theta$ solving the implicit scalar equation (24).

The study of the existence of three-dimensional weak solutions to the variational problem (18)-(19), under the assumption that the mapping $x \in$ $\Omega \mapsto \sigma(x, T)$ is discontinuous for every $T \in \mathbb{R}$, is still an open problem. When the coefficient $\sigma$ of the principal part of the divergence form elliptic equation (3) is a discontinuous function on the spatial variable, it invalidates the smoothness of the solution as is carried out in the literature [6, 24, 26, 29, 32].

\section{$3 \quad$ Auxiliary results}

The existence of a unique solution $\phi \in H^{1}(\Omega)$, such that $\int_{\partial \Omega} \phi d s=0$, to an auxiliary problem is consequence of Lax-Milgram lemma, for details see

Proposition 3.1 ([10, Theorem 4.1]) Let the assumptions (H2) and (12) or (15) be fulfilled. Assume that $n \geq 2, \Omega \in C^{0,1}, \xi \in H^{1}(\Omega)$ and $h \in L^{p}(\partial \Omega)$ verify (9) for $p>2(n-1) / n$. Then there exists a unique weak solution $\phi \in H^{1}(\Omega)$, such that $\int_{\partial \Omega} \phi d s=0$, to the variational problem

$$
\int_{\Omega} \sigma(\xi) \nabla \phi \cdot \nabla \eta d x=-\int_{\Omega} \sigma(\xi) \alpha(\xi) \nabla \xi \cdot \nabla \eta d x+\int_{\partial \Omega} h \eta d s,
$$

for all $\eta \in H^{1}(\Omega)$ and in particular for all $\eta \in W^{1, p^{\prime}}(\Omega)$.

Next, we establish some regularity for the potential auxiliary solution.

Proposition 3.2 Let $p>n, h \in L^{p}(\partial \Omega)$ verify (9)), $\xi \in W^{2, q}(\Omega)$ with $q=p n /(p+n)$, (H4) be fulfilled, and $\phi \in H^{1}(\Omega)$ solve the problem (26). If $\sigma: \Omega \times \mathbb{R} \rightarrow \mathbb{R}$ is a uniformly continuous function verifying (8), then $\nabla \phi \in \mathbf{L}^{p}(\Omega)$ and it verifies

$$
\|\nabla \phi\|_{p, \Omega} \leq C\left(n, p, \Omega, \sigma_{\#}, \sigma^{\#}\right)\left(\sigma^{\#} \alpha^{\#}\|\nabla \xi\|_{p, \Omega}+\|h\|_{p, \partial \Omega}\right) .
$$


Proof. For $p>n$, we have $q>n / 2$,

$$
\begin{array}{r}
\xi \in W^{2, q}(\Omega) \hookrightarrow C^{0,2-n / q}(\bar{\Omega}), \quad \alpha(\xi) \nabla \xi \in \mathbf{W}^{1, q}(\Omega) \hookrightarrow \mathbf{L}^{q(n-1) /(n-q)}(\partial \Omega), \\
\alpha(\xi) \nabla \xi \cdot \mathbf{n} \in L^{q(n-1) /(n-q)}(\partial \Omega) \equiv L^{p(n-1) / n}(\partial \Omega) \hookrightarrow W^{-1 / p, p}(\partial \Omega),
\end{array}
$$

for $\mathbf{n} \in \mathbf{L}^{\infty}(\partial \Omega)$. Moreover, $h / \sigma(\xi) \in W^{-1 / p, p}(\partial \Omega)$ and $\nabla \xi \in \mathbf{L}^{p}(\Omega)$ implies that $\nabla \cdot(\sigma(\xi) \alpha(\xi) \nabla \xi) \in\left(\mathbf{W}^{1, p^{\prime}}(\Omega)\right)^{\prime}$ for $p>n$. By appealing to the regularity theory [15, 19] for the solution $\phi \in H^{1}(\Omega)$ of the boundary value problem (in the sense of distributions)

$$
\begin{aligned}
& \nabla \cdot(\sigma(\xi) \nabla \phi+\sigma(\xi) \alpha(\xi) \nabla \xi)=0 \quad \text { in } \Omega \\
& \sigma(\xi)(\nabla \phi+\alpha(\xi) \nabla \xi) \cdot \mathbf{n}=h \quad \text { on } \partial \Omega,
\end{aligned}
$$

and observing that $\xi \in W^{1, p}(\Omega) \hookrightarrow C^{0,1-n / p}(\bar{\Omega})$ for $p>n$ warrants that $\sigma(\cdot, \xi)$ is uniformly continuous, then the regularity of weak solutions relative to $W^{1, p}(\Omega)$ and the estimate (27) arise.

The following result deals with the existence and uniqueness of a strong temperature auxiliary solution.

Proposition 3.3 Let $p>n, \xi \in W^{1, p}(\Omega)$, (H1) and (H3)-(H4) be fulfilled, and $\phi \in H^{1}(\Omega)$ solve the problem (26)). If $\sigma: \Omega \times \mathbb{R} \rightarrow \mathbb{R}$ is a uniformly continuous function verifying (8), then there exists a unique weak solution $\theta \in W^{2, p n /(p+n)}(\Omega)$ solving the problem, for all $\eta \in W^{1, p^{\prime}}(\Omega)$,

$$
k \int_{\Omega} \nabla \theta \cdot \nabla \eta d x+\int_{\partial \Omega} \alpha(\xi) h \theta \eta d s=\int_{\Omega}(F(\cdot, \xi, \nabla \xi, \nabla \phi)+g) \eta d x,
$$

with $F: \Omega \times \mathbb{R}^{2 n+1} \rightarrow \mathbb{R}$ defined as $F(x, T, \mathbf{a}, \mathbf{b})=$

$\sigma(x, T)\left(\alpha(x, T)\left(\alpha(x, T)+\frac{\partial \alpha}{\partial T}(x, T) T\right)|\mathbf{a}|^{2}+\left(2 \alpha(x, T)+\frac{\partial \alpha}{\partial T}(x, T) T\right) \mathbf{a} \cdot \mathbf{b}+|\mathbf{b}|^{2}\right)$.

Proof. The existence and uniqueness of $\phi \in W^{1, p}(\Omega)$ is consequence of Propositions 3.1 and 3.2. By appealing to the elliptic equations theory [19], from $F(\xi, \nabla \xi, \nabla \phi)+g \in L^{p / 2}(\Omega)$, the regularity theory for the Laplace equation in convex domains guarantees the existence of a unique solution $\theta \in W^{2, p n /(p+n)}(\Omega)$ of the Robin problem

$$
\begin{aligned}
& -k \Delta \theta=F(\cdot, \xi, \nabla \xi, \nabla \phi)+g \quad \text { in } \Omega ; \\
& k \nabla \theta \cdot \mathbf{n}+\alpha(\xi) h \theta=0 \quad \text { on } \partial \Omega,
\end{aligned}
$$


taking into account that the Korn perturbation method [19, pp. 107-109] can be adapted if the coefficient $\alpha(\cdot, \xi) h \in C(\partial \Omega)$ is such that the assumption (10) holds. For this, we observe that $\xi \in W^{1, p}(\Omega) \hookrightarrow C(\bar{\Omega})$ and we recall (H3)-(H4).

For the regularity of the potential auxiliary solution $\phi$ when it is the unique weak solution for Neumann problem to an elliptic second order equation in divergence form with bounded and measurable coefficient, we can prove the following result.

Proposition 3.4 If the assumptions of Proposition 3.1 are fulfilled with $p=$ 2 and $\Omega$ is convex, then there exists a constant $\epsilon>0$ such that the weak solution $\phi \in H^{1}(\Omega)$ of (26) belongs to $W^{1,2+\epsilon}(\Omega)$, i.e.

$$
\|\nabla \phi\|_{2+\epsilon, \Omega} \leq K_{2}\left(\sigma^{\#} \alpha^{\#}\|\nabla \xi\|_{2, \Omega}+\|h\|_{2, \partial \Omega}\right),
$$

with a constant $K_{2}>0$ only dependent on the data.

Proof. Denote the operator $A$ by

$$
\langle A \phi, \eta\rangle=\int_{\Omega} \sigma(\xi) \nabla \phi \cdot \nabla \eta d x
$$

Then $\phi \in H^{1}(\Omega)$ is a weak solution to the second order elliptic differential equation $A u=F$, under

$$
F=\nabla \cdot(\sigma(\xi) \alpha(\xi) \nabla \xi)+h \in\left(H^{1}(\Omega)\right)^{\prime} \hookrightarrow\left(W^{1, p}(\Omega)\right)^{\prime}, \quad \forall p \geq 2 .
$$

Since the boundedness property

$$
\sigma_{\#} \leq \sigma(\cdot, \xi) \leq \sigma^{\#} \text {, a.e. in } \Omega,
$$

is fulfilled, considering that $\xi \in L^{1}(\Omega)$ and the assumption (8) on $\sigma$ holds, then the Neumann version of the general result on the higher regularity for weak solutions to the mixed boundary value problems (cf. [20, Theorem 1], also [21]) guarantees that $\phi \in W^{1,2+\epsilon}(\Omega)$ for some $\epsilon>0$.

Although Proposition 3.4 is valid for any dimensional space $(n \geq 2)$, we only used it for $n=2$. Let us precise its application in the following proposition.

Proposition 3.5 Let $\xi \in W^{1,2+\epsilon}(\Omega)$, (H1)-(H4) be fulfilled, and $\phi \in H^{1}(\Omega)$ solve the problem (26). If the assumptions of Proposition 3.4 hold, then there exists a unique weak solution $\theta \in W^{2,2 p /(p+2)}(\Omega)$ solving (28) with $p=2+\epsilon$. 
PROOF. The imperative requirement of the embedding $W^{1,2+\epsilon}(\Omega) \hookrightarrow C(\bar{\Omega})$ yields that $\alpha(\cdot, \xi) h \in C(\partial \Omega)$ provided by (H3)-(H4). Thus, Proposition 3.4 ensures that the argument of the proof of Proposition 3.3 is still valid, concluding the claim.

\section{Proof of Theorem 2.1}

First we recall the Tychonoff extension to weak topologies of the Schauder fixed point theorem [16, pp. 453-456 and 470].

Theorem 4.1 Let $K$ be a nonempty compact convex subset of a locally convex space $X$. Let $\mathcal{L}: K \rightarrow K$ be a continuous operator. Then $\mathcal{L}$ has at least one fixed point.

If we provide any Banach space with the weak topology, every closed ball is convex and weakly sequential compact.

In order to apply Theorem 4.1, let us consider the operator $\mathcal{L}$ defined in a closed ball $\bar{B}_{R} \subset W^{2, p n /(p+n)}(\Omega)$ such that

$$
\mathcal{L}: \xi \in \bar{B}_{R} \mapsto \phi \mapsto \theta \in W^{2, p n /(p+n)}(\Omega),
$$

where $\phi \in W^{1, p}(\Omega)$ solves the problem (26) , for all $\eta \in W^{1, p^{\prime}}(\Omega)$, and $\theta$ solves the problem (28).

The existence of a unique solution $\phi \in W^{1, p}(\Omega)$, such that $\int_{\partial \Omega} \phi d s=0$, to the problem (26) is consequence of Propositions 3.1 and 3.2, and it verifies (27).

Hence, for $p>n$, we find $\theta \in W^{1, p}(\Omega)$ from Proposition 3.3, and the estimate

$\|\theta\|_{2, p n /(p+n), \Omega} \leq K\left(A\|\nabla \xi\|_{p, \Omega}^{2}+B\|\nabla \xi\|_{p, \Omega}\|\nabla \phi\|_{p, \Omega}+\sigma^{\#}\|\nabla \phi\|_{p, \Omega}^{2}+\|g\|_{p / 2, \Omega}\right)$,

is verified with $K$ denoting a constant dependent on $\Omega, n$ and $p, A$ and $B$ given by (23), and $\varkappa:=\min \left\{k, \alpha_{\#} h_{\#}\right\}+\alpha^{\#} h_{1} C_{1}>0$.

Thus $\mathcal{L}$ is well defined.

Next, let us prove that $\mathcal{L}\left(\bar{B}_{R}\right) \subset \bar{B}_{R}$. Let $\xi \in \bar{B}_{R}$ be arbitrary and $(\phi, \theta)$ be the corresponding solution solving (26) and (28). Thus (27) and (30) read

$$
\begin{array}{r}
\|\phi\|_{1, p, \Omega} \leq C\left(\sigma^{\#} \alpha^{\#} R+\|h\|_{p, \partial \Omega}\right) \\
\|\theta\|_{2, p n /(p+n), \Omega} \leq K\left(A R^{2}+B R\|\nabla \phi\|_{p, \Omega}+\sigma^{\#}\|\nabla \phi\|_{p, \Omega}^{2}+\|g\|_{p / 2, \Omega}\right),
\end{array}
$$


with $C=C\left(n, p, \Omega, \sigma_{\#}, \sigma^{\#}\right)$. Inserting (31) into (32) it follows

$$
\|\theta\|_{2, p n /(p+n), \Omega} \leq a_{2} R^{2}+a_{1} R+a_{0},
$$

where

$$
\begin{aligned}
& a_{2}=K \sigma^{\#} \alpha^{\#}\left(1+C \sigma^{\#}\right)\left(\alpha^{\#}\left(2+C \sigma^{\#}\right)+\mu^{\#}\right) ; \\
& a_{1}=K C \sigma^{\#}\left(2 \alpha^{\#}\left(1+\sigma^{\#}\right)+\mu^{\#}\right)\|h\|_{p, \partial \Omega} ; \\
& a_{0}=K\left(C^{2} \sigma^{\#}\|h\|_{p, \partial \Omega}^{2}+\|g\|_{p / 2, \Omega}\right) .
\end{aligned}
$$

Therefore, $\mathcal{L}(\xi)=\theta \in \bar{B}_{R}$ if and only if $a_{2} R^{2}+\left(a_{1}-1\right) R+a_{0} \leq 0$, i.e. for instance if the smallness condition

$$
a_{1}<1 \text { and } 4 a_{0} a_{2}<\left(1-a_{1}\right)^{2}
$$

is assumed.

\subsection{The weak sequential continuity of $\mathcal{L}$}

Let $\left\{\xi_{m}\right\}_{m \in \mathbb{N}}$ be a sequence in $\bar{B}_{R}$ verifying

$$
\xi_{m} \rightarrow \xi \quad \text { in } W^{2, p n /(p+n)}(\Omega) \hookrightarrow \hookrightarrow W^{1, p}(\Omega),
$$

and $\left(\phi_{m}, \theta_{m}\right)$ is the correspondent solution to (26) and (28), for each $m \in \mathbb{N}$. From the estimates (27) and (30) we can extract a subsequence, still labeled by $\left(\phi_{m}, \theta_{m}\right)$, such that

$$
\phi_{m} \rightarrow \phi \quad \text { in } W^{1, p}(\Omega), \quad \theta_{m} \rightarrow \theta \quad \text { in } W^{2, p n /(p+n)}(\Omega) \hookrightarrow \hookrightarrow W^{1, p}(\Omega) .
$$

Thanks to Remark 2.2 it follows

$$
\xi_{m} \rightarrow \xi, \quad \theta_{m} \rightarrow \theta, \quad \phi_{m} \rightarrow \phi \quad \text { in } C^{0,1-n / p}(\bar{\Omega}) .
$$

In particular, $\int_{\partial \Omega} \phi_{m} d s=0 \rightarrow \int_{\partial \Omega} \phi d s=0$. By the continuity of the Nemytskii operators $\alpha$ and $\sigma$, we can pass to the limit in $(26) m$ as $m$ tends to infinity, concluding that $\phi \in W^{1, p}(\Omega)$ is the limit solution, i.e. it verifies (26)).

In the sequel, let us pass to the limit in $(28)_{m}$ as $m$ tends to infinity. First, the mapping $\xi \in L^{1}(\Omega) \mapsto \alpha(\xi) \in L^{r}(\Omega)$, for all $r<+\infty$, is continuous by (H4), thus the passage to the limit to the left hand side of (28) is 
straightforward. In order to study the RHS, we define

$$
\begin{aligned}
I_{1, m} & =\int_{\Omega} \sigma\left(\xi_{m}\right) \alpha\left(\xi_{m}\right)\left(\alpha\left(\xi_{m}\right)+\frac{\partial \alpha}{\partial T}\left(\xi_{m}\right) \xi_{m}\right)\left|\nabla \xi_{m}\right|^{2} \eta d x \\
I_{2, m} & =\int_{\Omega} \sigma\left(\xi_{m}\right)\left(2 \alpha\left(\xi_{m}\right)+\frac{\partial \alpha}{\partial T}\left(\xi_{m}\right) \xi_{m}\right) \nabla \xi_{m} \cdot \nabla \phi_{m} \eta d x \\
I_{3, m} & =\int_{\Omega} \sigma\left(\xi_{m}\right)\left|\nabla \phi_{m}\right|^{2} \eta d x .
\end{aligned}
$$

Recalling Remark 2.2 we get $\eta \in W^{1, p^{\prime}}(\Omega) \hookrightarrow L^{p n /(p n-n-p)}(\Omega) \hookrightarrow L^{p /(p-2)}(\Omega)$ for $p>n$.

From (34) we have $\left|\nabla \xi_{m}\right|^{2} \rightarrow|\nabla \xi|^{2}$ in $L^{p / 2}(\Omega)$. Considering that the mapping $\xi \in L^{1}(\Omega) \mapsto \sigma(\xi) \alpha^{2}(\xi) \eta \in L^{p /(p-2)}(\Omega)$ is continuous thus the first term in $I_{1, m}$ passes to the limit as $m$ tends to infinity. Using (34) and (35) we have $\xi_{m}\left|\nabla \xi_{m}\right|^{2} \rightarrow \xi|\nabla \xi|^{2}$ in $L^{p / 2}(\Omega)$. Considering that the mapping $\xi \in L^{1}(\Omega) \mapsto \sigma(\xi) \alpha(\xi) \frac{\partial \alpha}{\partial T}(\xi) \eta \in L^{p /(p-2)}(\Omega)$ is continuous thus the second term in $I_{1, m}$ passes to the limit as $m$ tends to infinity.

Analogously, we take to the limit in $I_{2, m}$ observing that the strong-weak convergence product $\nabla \xi_{m} \cdot \nabla \phi_{m} \rightarrow \nabla \xi \cdot \nabla \phi$ holds in $L^{p / 2}(\Omega)$.

In order to be in conditions for finding that $\theta$ is a limit solution, let us prove the continuity of the solution mapping $\xi \in W^{1, p}(\Omega) \mapsto \phi=\phi(\xi) \in$ $W^{1, s}(\Omega)$ in the strong topology for $s=2 p n /(p+n)<p$. Take the difference of (26) $)_{m}$ and (26) verified by the solutions $\phi_{m}$ and $\phi$, respectively, and choose $\eta=\phi_{m}-\phi$ as a test function. Thus, it results

$$
\begin{array}{r}
\sigma_{\#}\left\|\nabla\left(\phi_{m}-\phi\right)\right\|_{2, \Omega}^{2} \leq \int_{\Omega}\left(\sigma(\xi)-\sigma\left(\xi_{m}\right)\right) \nabla \phi \cdot \nabla\left(\phi_{m}-\phi\right) d x+ \\
+\int_{\Omega}\left(\sigma(\xi) \alpha(\xi) \nabla \xi-\sigma\left(\xi_{m}\right) \alpha\left(\xi_{m}\right) \nabla \xi_{m}\right) \cdot \nabla\left(\phi_{m}-\phi\right) d x \longrightarrow 0, \quad \text { as } m \rightarrow \infty .
\end{array}
$$

Then, we conclude that $\nabla \phi_{m} \rightarrow \nabla \phi$ in $\mathbf{L}^{2}(\Omega)$, and consequently $\nabla \phi_{m} \rightarrow \nabla \phi$ a.e. in $\Omega$ and $\left|\nabla \phi_{m}\right|^{2} \rightarrow|\nabla \phi|^{2}$ in $L^{s / 2}(\Omega) \hookrightarrow L^{p n /(p+n)}(\Omega)$. Thus $I_{3, m}$ passes to the limit as $m$ tends to infinity, concluding the proof of weak continuity of the operator $\mathcal{L}$.

Then the Schauder fixed point theorem can be used and it guarantees the existence of $(\theta, \phi)$ in the conditions to Theorem 2.1. 


\subsection{The validation of the estimates $(20)-(22)$}

Let $(\theta, \phi) \in W^{2, p n /(p+n)}(\Omega) \times W^{1, p}(\Omega)$ be a weak solution to the variational problem (18)-(19)

Choose $\eta=\phi \in W^{1, p}(\Omega)$ as a test function in (19). Using (8) , the upper bound of $|\alpha|$ and the Sobolev-Poincaré inequality then (20) holds.

From the regularity theory for the Robin-Laplace problem and by virtue of the existence of a solution $\theta \in W^{2, p n /(p+n)}(\Omega)$ we proceed as in (30) now for $k>\alpha_{\#} h_{\#}$ resulting the estimate

$$
\|\nabla \theta\|_{p, \Omega} \leq K\left(A\|\nabla \theta\|_{p, \Omega}^{2}+B\|\nabla \theta\|_{p, \Omega}\|\nabla \phi\|_{p, \Omega}+\sigma^{\#}\|\nabla \phi\|_{p, \Omega}^{2}+\|g\|_{p / 2, \Omega}\right),
$$

with $K$ denoting a constant independent on $k$. Combining this result with the estimate (27) with $\xi$ replaced by $\theta$ and using (33) we conclude (21).

Choose $\eta=\theta \in W^{1, p}(\Omega)$ as a test function in (18). Then applying the Hölder inequality and using the assumptions (H1)-(H4) it follows

$$
\begin{array}{r}
k\|\nabla \theta\|_{2, \Omega}^{2} \leq \alpha^{\#} h_{1}\|\theta\|_{2, \Sigma}^{2}+\left(A\|\nabla \theta\|_{2, \Omega}^{2}+B\|\nabla \theta\|_{2, \Omega}\|\nabla \phi\|_{2, \Omega}+\right. \\
\left.+\sigma^{\#}\|\nabla \phi\|_{2, \Omega}^{2}+\|g\|_{1, \Omega}\right)\|\theta\|_{\infty, \Omega} .
\end{array}
$$

This yields the estimate (22).

\section{Proof of Theorem 2.2}

For each given $k>0$, let $\left(\theta_{k}, \phi_{k}\right)$ be a solution to (18)-(19) in accordance with Theorem [2.1. From estimates (22) and (20) there exist subsequences still denoted by $\theta_{k}$ and $\phi_{k}$ such that, for $k \rightarrow+\infty$,

$$
\begin{aligned}
\nabla \theta_{k} \rightarrow 0 \quad \text { in } \mathbf{L}^{2}(\Omega) ; \quad & \theta_{k} \rightarrow \Theta \quad \text { in } W^{1, p}(\Omega) \hookrightarrow \hookrightarrow C(\bar{\Omega}) ; \\
& \phi_{k} \rightarrow \phi \quad \text { in } H^{1}(\Omega),
\end{aligned}
$$

with $\Theta$ constant on $\bar{\Omega}$. Hence we can pass to the limit in (19) as $k$ tends to infinity resulting

$$
\int_{\Omega} \sigma(\cdot, \Theta) \nabla \phi \cdot \nabla \eta d x=\int_{\partial \Omega} h \eta d s, \quad \forall \eta \in W^{1, p^{\prime}}(\Omega),
$$

or equivalently (6) $-($ (7) $)$. 
In particular, if we take $\eta=1$ in (18) we can pass to the limit as $k$ tends to infinity resulting (24).

Using (8) it follows

$$
\sigma_{\#} \int_{\Omega}|\nabla \phi|^{2} d x+\int_{\Omega} g d x \leq \Theta \int_{\partial \Omega} \alpha(\cdot, \Theta) h d s \leq \sigma^{\#} \int_{\Omega}|\nabla \phi|^{2} d x+\int_{\Omega} g d x .
$$

Taking into account that the assumption $(\mathrm{H} 4)_{+}$or $(\mathrm{H} 4)_{-}$and also (17) imply

$$
\begin{aligned}
& \int_{\partial \Omega} \alpha(\cdot, \Theta) h d s \leq \alpha^{\#} \int_{\partial \Omega}|h| d s \\
& \int_{\partial \Omega} \alpha(\cdot, \Theta) h d s \geq \alpha_{\#} h_{\#}|\Gamma|+\alpha^{\#} h_{1}|\Sigma|>0,
\end{aligned}
$$

we derive (25), concluding the proof of Theorem 2.2 .

\section{The two-dimensional limit model}

\subsection{Proof of Theorem 2.3}

Arguing as in Theorem 2.1, the Schauder fixed point argument can be applied. For this, it is sufficient to see that the regularity relative to $W_{\text {loc }}^{1, p}(\bar{\Omega})=$ $W^{1, p}(\Omega)$ ( $\Omega$ bounded) can be applied for the unique weak solution of the variational problem (26) for $p=2+\epsilon>2=n$ in accordance to Proposition 3.4. Thus Proposition 3.5 guarantees the existence of $\theta \in W^{2,2 p /(p+2)}(\Omega)$ verifying (30). For every $\xi \in \bar{B}_{R}$, inserting (29) into (30) it follows

$$
\begin{aligned}
\|\theta\|_{2,2 p /(p+2), \Omega} \leq & K\left(A R^{2}+B R K_{2}\left(\sigma^{\#} \alpha^{\#} R+\|h\|_{2, \partial \Omega}\right)+\right. \\
& \left.+\sigma^{\#} K_{2}{ }^{2}\left(\sigma^{\#} \alpha^{\#} R+\|h\|_{2, \partial \Omega}\right)^{2}+\|g\|_{p / 2, \Omega}\right) .
\end{aligned}
$$

Next arguing as in Section 4 it leads to a smallness condition. From the continuity of the Nemytskii operator $\sigma$ due to the Krasnoselski Theorem we can proceed as in Section 4.1, considering that $I_{3, m}$ passes to the limit as $m$ tends to infinity since

$$
\begin{array}{r}
\sigma\left(\xi_{m}\right) \rightarrow \sigma(\xi) \quad \text { weakly* in } L^{\infty}(\Omega) ; \\
\left|\nabla \phi_{m}\right|^{2} \eta \rightarrow|\nabla \phi|^{2} \eta \quad \text { in } L^{1}(\Omega) .
\end{array}
$$

This concludes that we are in the conditions of applying Theorem 4.1. 


\subsection{Proof of Proposition 2.1}

We can proceed as in Section 5 considering the existence of the sequence of solutions is provided by Theorem 2.3 and the proposition follows.

\section{References}

[1] S.N. Antontsev and M. Chipot, The thermistor problem: Existence, smoothness, uniqueness, blow up, SIAM J. Math. Anal. 25 (1994), 6783.

[2] M. Baddi, Existence of periodic solutions for the thermistor problem with the Joule-Thomson effect, Ann. Univ. Ferrara 54 (2008), 1-10.

[3] A. Bulusu and D.G. Walker, Review of electronic transport models for thermoelectric materials, Superlattices and Microstructures 44 (2008), $1-36$.

[4] S.-S. Byun and L. Wang, Gradient estimates for elliptic systems in nonsmooth domains, Math. Ann. 341 (2008), 629-650.

[5] A. Chakraborty, B.B. Saha, S. Koyama and K.C. Ng, Thermodynamic modelling of a solid state thermoelectric cooling device: Temperatureentropy analysis, Internat. J. of Heat and Mass Transfer 49 (2006), 3547-3554.

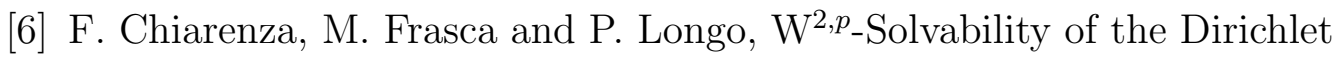
Problem for Nondivergence Elliptic Equations with VMO Coefficients, Transactions of the American Mathematical Society 336 :2 (1993), 841853.

[7] M. Chipot and J.F. Rodrigues, On a class of nonlocal nonlinear elliptic problems, RAIRO Modél. Math. Anal. Numér. 26 :3 (1992), 447-467.

[8] G. Cimatti, Existence and uniqueness for the equations of the JouleThomson effect, Appl. Anal. 41 (1991), 131-144.

[9] G. Cimatti, Stability and multiplicity of solutions for the thermistor problem, Annali di Matematica 181 (2002), 181-212. 
[10] L. Consiglieri, The Joule-Thomson effect on the thermoelectric conductors, Z. Angew. Math. Mech. 89 :3 (2009), 218-236.

[11] L. Consiglieri and M.C. Muñiz, Existence of a solution for a free boundary problem in the thermoelectrical modelling of an aluminium electrolytic cell, European Journal of Applied Mathematics 14 (2003), 201216.

[12] M. Costabel, M. Dauge and S. Nicaise, Singularities of Maxwell interface problems, M2AN 33 :3 (1999), 627-649.

[13] B.E.J. Dahlberg and C.E. Kenig, Hardy Spaces and the Neumann Problem in $L^{p}$ for Laplace's Equation in Lipschitz Domains, The Annals of Mathematics, Second Series 125 :3 (1987), 437-465.

[14] M. Dauge, Neumann and mixed problems on curvilinear polyhedra, Integr. Equat. Oper. Th. 15 (1992), 227-261.

[15] R. Dautray and J.L. Lions, Mathematical analysis and numerical methods for science and technology, Physical origins and classical methods. Vol. 1, Springer-Verlag, Berlin-Heidelberg 1990.

[16] N. Dunford and J.T. Schwartz, Linear operators. Part I, Interscience Publ., NY 1958.

[17] J. Elschner, J. Rehberg and G. Schmidt, Optimal regularity for elliptic transmission problems including $\mathrm{C} 1$ interfaces, Interfaces Free Bound. 9 :2 (2007), 233-252.

[18] J.-G. Gasser, Understanding the resistivity and absolute thermoelectric power of disordered metals and alloys, J. Phys.: Condens. Matter 20 (2008) 114103 (14pp)

[19] P. Grisvard, Elliptic problems in nonsmooth domains, Monographs and studies in mathematics 24, Pitman, Boston 1985.

[20] K. Groger, A $W^{1, p}$ - estimate for solutions to mixed boundary value problems for second order elliptic differential equations, Mathematische Annalen 283 (1989), 679-687. 
[21] K. Groger and J..Rehberg, Resolvent estimates in $W^{-1, p}$ for second order elliptic differential operators in case of mixed boundary conditions, Mathematische Annalen 285 (1989), 105-113.

[22] A.F. Ioffe, Physics of semiconductors, Academic Press Inc., New York 1960 .

[23] D.V. Keller, Jr. and G. Maxwell, Physio-chemical factors contributing to thermoelectric phenomena. Part 1: Thomson heat in metallic systems, Syracuse Univ. Research Inst., NY 1965.

[24] C.E. Kenig and J. Pipher, The Neumann problem for elliptic equations with non-smooth coefficients, Invent. math. 113 (1993), 447-509.

[25] M. Jakob, Heat transfer. Vol. I, Wiley, New York 1949.

[26] W. Littman, G. Stampacchia and H.F. Weinberger, Regular points for elliptic equations with discontinuous coefficients, Ann. Scuola Norm. Sup. Pisa 17 :1-2 (1963), 43-77.

[27] D.K.C. MacDonald, Thermoelectricity: an introduction to the principles, John Wiley \& Sons, Inc., New York 1962.

[28] D. Medková, The Neumann problem for the Laplace equation on general domains, Czechoslovak Mathematical Journal 57 :132 (2007), 1107-1139.

[29] N.G. Meyers, An $L^{p}$-estimates for the gradient of solutions of second order elliptic divergence equations, Ann. Scuola Norm. Sup. Pisa 17 (1963), 189-206.

[30] M.T.G. Montesinos and F.O. Gallego, On certain doubly non-uniformly and singular non-uniformly elliptic systems, Nonlinear Analysis $\mathbf{5 4}$ (2003), 1193-1204.

[31] Y. Nishiura, Global branching theorem for spatial patterns of a reactiondiffusion system, Proc. Japan Acad. Ser. A Math. Sci. 55 :6 (1979), 201-204.

[32] M.A. Ragusa, Linear growth coefficients in quasilinear equations, Nonlinear Differ. Equ. Appl. NoDEA 13 (2007), 605-617. 
[33] X. Xu, Existence and uniqueness for the nonstationary problem of the electrical heating of a conductor due to the Joule-Thomson effect, Internat. J. Math. Math. Sci. 16 :1 (1993), 125-138.

[34] X. Xu, Local regularity theorems for the stationary thermistor problem with oscillating degeneracy, Journal of Mathematical Analysis and Applications 338 :1 (2008), 274-284.

[35] O. Yamashita, Effect of temperature dependence of electrical resistivity on the cooling performance of a single thermoelectric element, Applied Energy 85 (2008), 1002-1014.

[36] D. Zanger, The inhomogeneous Neumann problem in Lipschitz domains, Comm. Partial Differential Equations 25 (2000), 1771-1808. 